Revue des patrimoines

$40 \mid 2019$

Lits historiques. Première anthologie des lits européens du XVe au XIXe siècle

\title{
Naître, aimer, mourir. Les lits (et leurs habitants) dans la peinture bolonaise des $\mathrm{XVI}^{\mathrm{e}}$ et $\mathrm{XVII}^{\mathrm{e}}$ siècles
}

Being Born, Loving and Dying; Beds (and their Occupants) in the Painting of Bologna during the Sixteenth and Seventeenth centuries

\section{Michele Danieli}

\section{OpenEdition}

Journals

Édition électronique

URL : http://journals.openedition.org/insitu/22782

DOI : 10.4000/insitu.22782

ISSN : $1630-7305$

Éditeur

Ministère de la Culture

Référence électronique

Michele Danieli, « Naître, aimer, mourir. Les lits (et leurs habitants) dans la peinture bolonaise des $x v{ }^{\mathrm{e}}$ et $x v \|^{e}$ siècles », In Situ [En ligne], 40 | 2019, mis en ligne le 23 septembre 2019, consulté le 14 novembre 2019. URL : http://journals.openedition.org/insitu/22782 ; DOI : 10.4000/insitu.22782

Ce document a été généré automatiquement le 14 novembre 2019.

\section{c) $(1) \odot$}

In Situ Revues des patrimoines est mis à disposition selon les termes de la licence Creative Commons Attribution - Pas d'Utilisation Commerciale - Pas de Modification 4.0 International. 


\title{
Naître, aimer, mourir. Les lits (et leurs habitants) dans la peinture bolonaise des $\mathrm{XVI}^{\mathrm{e}}$ et $\mathrm{XVII}{ }^{\mathrm{e}}$ siècles
}

\author{
Being Born, Loving and Dying; Beds (and their Occupants) in the Painting of \\ Bologna during the Sixteenth and Seventeenth centuries
}

Michele Danieli

La traduction de cet article a été réalisée par Marie Caillat.

Et ie ne voyois point qu'on m'estimast inferieur a mes condisciples, bien qu'il en eust desia entre eux quelques uns, qu'on destinoit a remplir les places des nos

maistres.

René Descartes, Discours de la méthode, 1637,

Première partie

1 Les études récentes comme les contributions rassemblées dans ce numéro ont mis en évidence les diverses significations qui s'entrecroisent à propos du lit, qui n'est pas seulement un élément du mobilier de la demeure à l'époque moderne mais aussi un espace à la fois physique et symbolique ${ }^{1}$. La double nature de cet objet trouve une parfaite incarnation dans la peinture du Xvi ${ }^{\mathrm{e}}$ siècle : en effet, le lit se transforme ici en un décor tout autant qu'en une scène de théâtre sur laquelle se déroulent des drames et des tragédies, des aventures héroïques et amoureuses.

2 Le petit corpus de peintures que je souhaite présenter ne comprend que des œuvres produites à Bologne entre la fin $\mathrm{du} \mathrm{xvI}^{\mathrm{e}}$ et le début du XvII siècle. Il s'agit donc d'une sélection très circonscrite dans le temps et dans l'espace (bien qu'elle représente potentiellement l'embryon d'une recherche plus vaste et systématique), qui, précisément parce qu'elle est restreinte à des limites aussi étroites, illustre bien les diverses formes et significations des lits figurés et représentés. 
Il est nécessaire de faire deux remarques liminaires. Je n'ai pas pris en compte ici de tableaux sur le thème de l'Annonciation, de la naissance de la Vierge ou de la naissance de saint Jean-Baptiste, car dans ces représentations le mobilier répond presque toujours à des exigences iconographiques de sobriété qui confèrent aux intérieurs domestiques un caractère anonyme et stéréotypé peu pertinent dans le cadre de notre propos $^{2}$. En second lieu, il faut rappeler qu'il n'existe à Bologne presque aucun lieu consacré à l'exercice du pouvoir. Jusqu'à la fin du xiII ${ }^{\mathrm{e}}$ siècle, la ville fut soumise à l'autorité du pape ; à partir de 1466, Giovanni II Bentivoglio tenta d'imposer le pouvoir de sa famille mais en 1506, à la suite de pressions du pape Jules II, sa famille fut chassée et la population détruisit son palais - la domus magna, le centre de son activité de commanditaire et l'édifice le plus proche d'un palais royal Renaissance que Bologne ait jamais abrité. Depuis cette date, et jusqu'aux campagnes napoléoniennes de 1796, Bologne fit partie des États pontificaux, gouvernée par un cardinal légat nommé par Rome et renouvelé tous les cinq ans. Il ne restait plus aux familles nobles qu'à se répartir les quarante sièges du Sénat, dont le nombre fut élevé à cinquante en 1589 , précisément pour maintenir la situation à l'équilibre et étouffer toute tentation d'hégémonie. Tout ceci pour dire que bien que Bologne fût un centre de production culturelle et artistique de première importance, elle ne connut jamais le faste d'une civilisation de cour et on n'y produisit jamais non plus aucun objet dévolu à la représentation du prestige politico-culturel d'un souverain: ni couronnes, ni résidences, ni, naturellement, aucun lit royal.

Le premier tableau que je voudrais étudier, Mercure ordonne à Énée d'abandonner Didon, fut exécuté par Orazio Samacchini (1532-1577) vers 1570, et est conservé au musée du Louvre $^{3}$ (fig. 1). Le récit qu'en donne le texte de l'Énéide diverge totalement de ce que l'on voit dans cette image : alors qu'il vient de rejoindre Carthage par les airs, Mercure aperçoit Énée en train de tracer des rues et des fortifications, portant une épée ornée de jaspe à la ceinture et un manteau de pourpre sur les épaules ${ }^{4}$. Samacchini situe au contraire l'épisode dans une chambre, dont la porte donne accès à une grande loggia. Énée, avec l'aide d'un putto, est en train de se dévêtir pour rejoindre Didon alors que Mercure fait son apparition pour lui ordonner d'abandonner l'Afrique. 
Figure 1

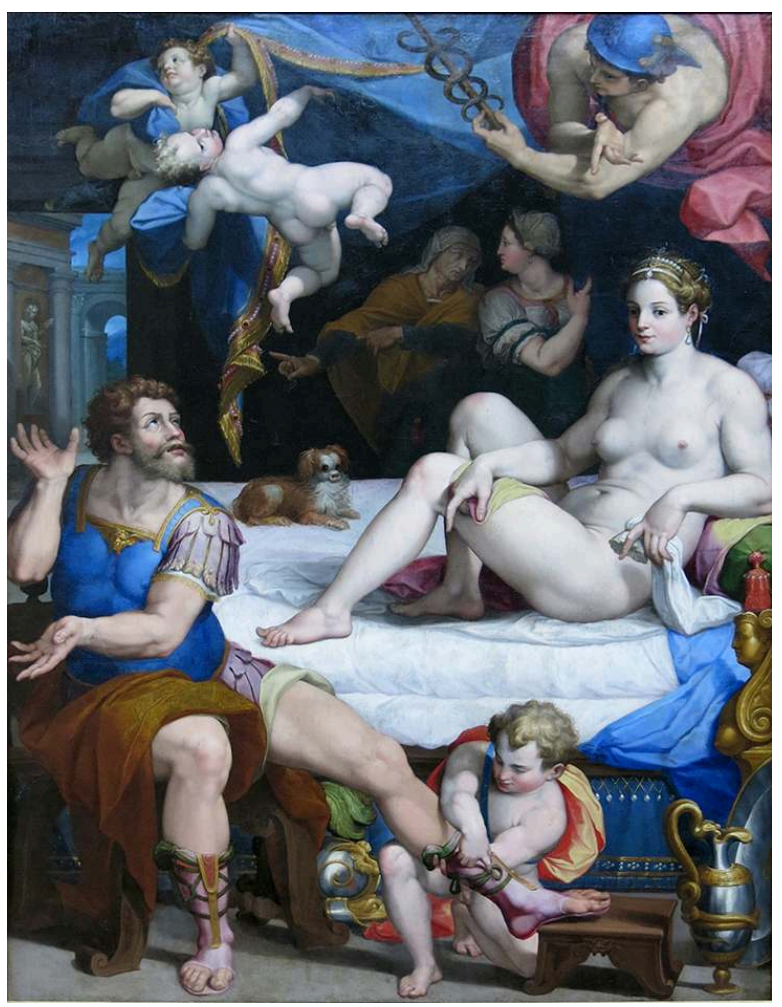

Orazio Samacchini, Mercure ordonne à Énée d'abandonner Didon. Paris, musée du Louvre.

Phot. RMN-Louvre. (c) RMN-Louvre.

5 Cette grande différence iconographique dérive certainement d'un tableau de Daniele da Volterra (1509-1566) exécuté pour Giovanni della Casa, décrit par Vasari : «alors qu'Énée se dévêt pour aller dormir avec Didon, il est surpris par Mercure ». La peinture de Daniele da Volterra est aujourd'hui perdue mais on connaît sa composition grâce à une copie et quelques dessins, et elle est plutôt similaire à celle de Samacchini ${ }^{5}$. Dans le tableau du Louvre, Didon apparait assise sur un lit luxueux, digne d'une reine. Pourtant ce lit est presque totalement caché, de sorte que nous ne pourrions pas en décrire la structure. Plus que représenté, le luxe est ici suggéré par le double matelas, les franges dorées du ciel de lit (ou bien s'agit-il de rideaux?) soulevé par les putti, et les riches broderies du tour de lit.

6 Plusieurs iconographies viennent se fondre dans l'image créée par Samacchini : outre l'épisode virgilien (très rarement représenté au Xvi ${ }^{e}$ siècle), il me semble que le geste du putto qui manie la chaussure d'Énée reprend un autre thème nuptial, celui de l'épisode d'Alexandre et Roxane. La fortune iconographique du sujet, on le sait, est liée à la description que Lucien de Samosate offrit de l'œuvre du peintre Aétion, laquelle rappelle explicitement le détail du putto défaisant la sandale de Roxane ${ }^{6}$. Ici les rôles sont inversés, et on ne comprend même pas très clairement si Énée est en train de s'habiller ou de se dévêtir ; je crois néanmoins que le putto endosse la même fonction que ceux qui, dans la description de Lucien, jouaient avec les armes d'Énée: «ils rappellent les goûts guerriers d'Alexandre qui, malgré sa passion pour Roxane, n'a point oublié celle des armes ${ }^{7}$.»

7 Juste après les deux personnages principaux, le protagoniste le plus important est véritablement le lit, qui occupe quasiment tout l'espace de la chambre, se déployant en 
profondeur. Au-delà du caractère nuptial de la couche, ses dimensions imposantes en font un lit de parade où la reine passe sa journée, coiffée avec grand soin, assistée de ses servantes, accompagnée, enfin, d'un petit chien qui ne peut symboliser ici, comme à l'habitude, la fidélité : aussi bien Énée que Didon sont, en quelque sorte, adultères, puisque leur amour trahit la mémoire de leurs époux défunts respectifs. Et c'est dans ce conflit intérieur que réside, pour une grande part, le drame. La dimension, pour ainsi dire " publique ", du lit accentue le caractère érotique de la nudité de Didon, si explicite qu'elle attira la censure des restaurateurs du XIX ${ }^{e}$ siècle qui en occultèrent le corps sous un improbable drapé de couleur ocres.

Dans le Suicide de Lucrèce de Denis Calvaert (1540-1619), c'est un lit tout à fait différent qui apparaît (fig. 2). L'œuvre du peintre d'Anvers (mais qui fit presque toute sa carrière à Bologne), conservée au musée Magnin de Dijon, appartient à ses dernières années d'activité, et doit sans doute être datée des premières années du Seicento, période où l'artiste se consacre plus fréquemment à la représentation de grands nus féminins9 .

Figure 2

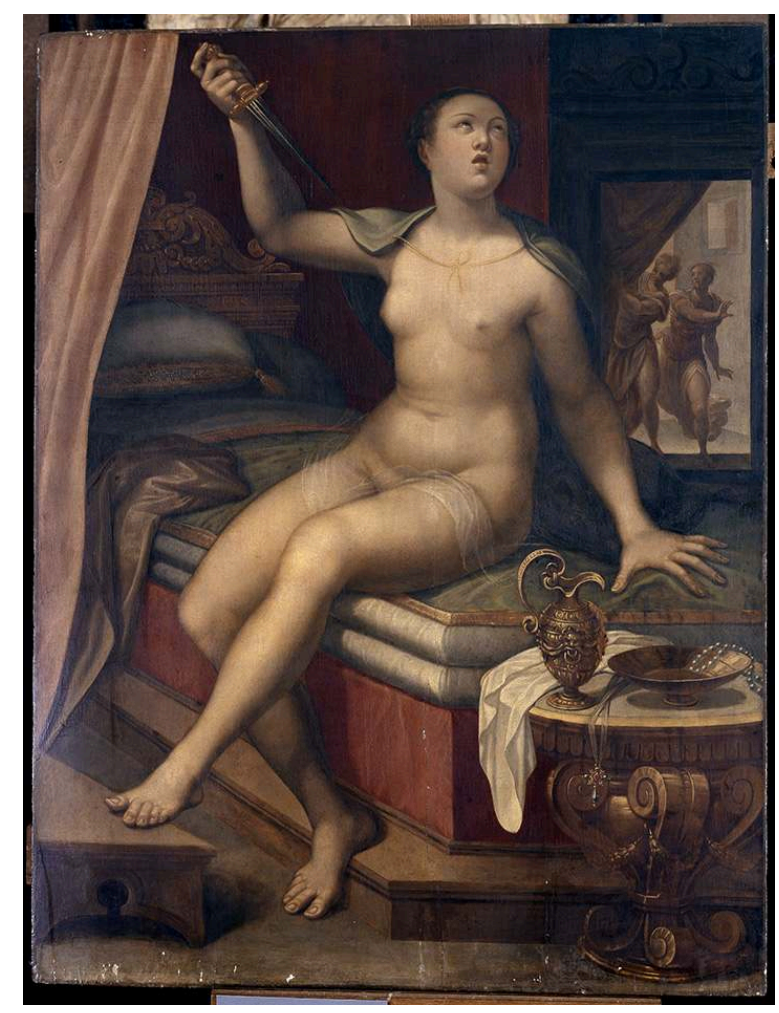

Denis Calvaert, Suicide de Lucrèce. Dijon, musée Magnin.

Phot. Franck Raux. (C) RMN-Grand Palais (musée Magnin).

Par rapport à celui de Samacchini, le lit représenté par Calvaert est beaucoup plus petit, peut-être même légèrement disproportionné car c'est au personnage que l'artiste a voulu conférer une plus grande monumentalité. En outre, son aspect étroit évoque une dimension tout à fait intime : les rideaux semblent en effet avoir été entr'ouverts juste pour nous permettre d'observer la scène.

Ce tableau est connu depuis longtemps mais le contexte de cette étude conduit à observer tout particulièrement le choix opéré par le peintre, qui assoit Lucrèce sur un lit véritablement démodé. Une couverture verte à simple bordure couvre deux matelas 
plutôt fins ; les rideaux et le tour de lit rouges ne présentent aucun décor. Seul élément remarquable, la tête de lit est ornée d'un motif de rinceaux végétaux. Dans l'ensemble, il s'agit donc d'un meuble modeste, moins raffiné par exemple que la carafe au décor ciselé ou la petite table placée au premier plan. Autour de la base du lit court un gradin qui semble évoquer les coffres-bancs qui encadraient les lits au $\mathrm{xv}^{\mathrm{e}}$ siècle: pensons notamment au célèbre lit du palais Davanzati (Florence) conservé au Metropolitan Museum of Art de New York ${ }^{10}$, malgré les doutes concernant sa véritable datation.

On a le sentiment que l'artiste a choisi de représenter un lit à la manière antique, non pas vraiment pour situer l'épisode à une époque révolue, désormais très lointaine et hors de portée (la Rome de la fin du vie siècle avant J.-C.), mais pour mettre en valeur la fermeté et la rigueur morale de la chaste Lucrèce. Du reste, Calvaert démontre, dans d'autres tableaux contemporains de celui-ci, sa connaissance de la mode en cours à l'époque: par exemple, dans un tableau d'une collection privée, le lit sur lequel Cléopâtre, plus élégante, décide de s'ôter la vie est plus moderne et raffiné. Le lit qui apparaît dans une belle peinture sur cuivre, Jupiter et Sémélé (fig. 3), est riche et orné : et on observe que les draps y sont en désordre puisque l'épisode fatal, selon le récit d'Ovide, se déroule pendant une rencontre amoureuse entre la jeune femme et le dieu. $\mathrm{Au}$ contraire, le lit de Lucrèce est intact, bien que la violence sexuelle y ait été consommée ${ }^{11}$.

Figure 3

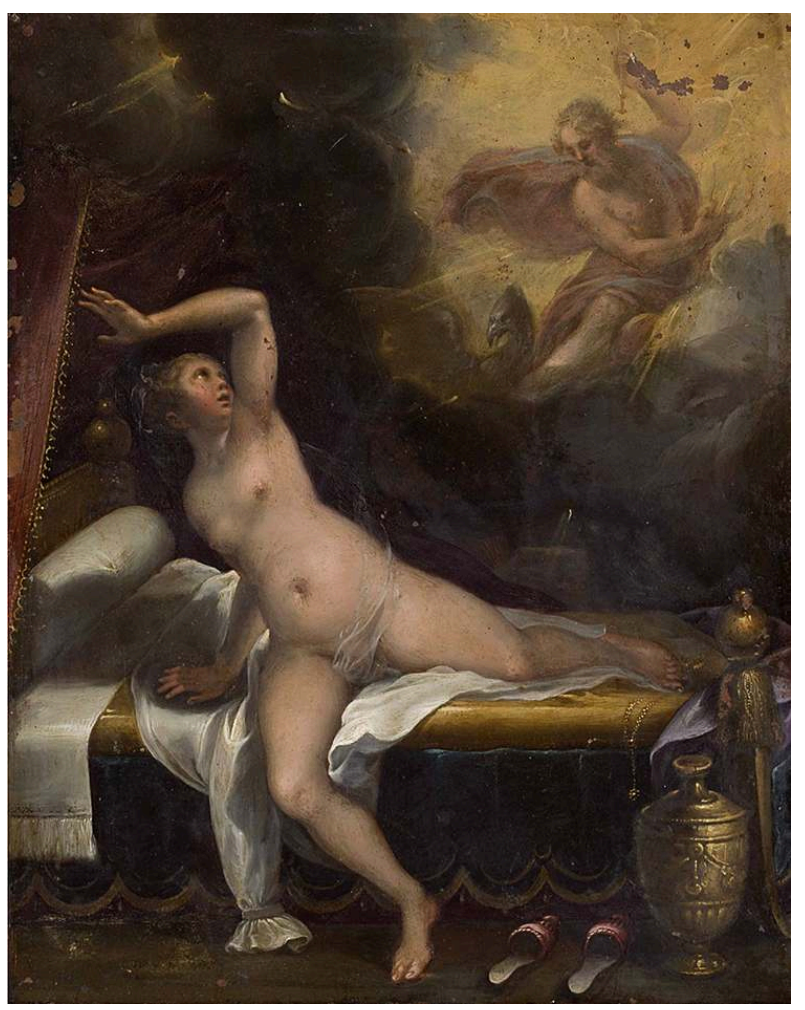

Denis Calvaert, Jupiter et Sémélé.

Phot. Michele Danieli. Collection particulière.

12 L'attitude de l'artiste, qui se sert du mobilier également pour conférer le juste registre narratif à la scène, doit nous mettre en garde quant à la précision des sources visuelles dans la description des objets de l'époque, et doit nous préserver de la tentation de les 
considérer comme des ready-made contemporains de l'image dans laquelle ils auraient été placés de manière presque fortuite.

Le lit figuré dans une peinture de Lorenzo Sabatini conservée à la Galleria Sabauda de Turin ne possède pas une véritable fonction d'ameublement mais sert à évoquer symboliquement un contexte matrimonial et devient un attribut iconographique fondamental d'une représentation allégorique (fig. 4). Alors attribué à Pellegrino Tibaldi (1527-1596), le tableau fut acquis autour de 1725 par le prince Eugène de Savoie et fut exposé dans la galerie de sa résidence viennoise, aujourd'hui connue sous le nom de palais du Belvédère. Dans un inventaire de 1736, son appartenance à l'école bolonaise était déjà oubliée, et il était attribué au florentin Francesco Salviati (1510-1563) : «Une femme tenant dans une main un miroir et dans l'autre un compas, qui représente la géométrie, de Salviati. » Bien qu'erronée, cette attribution à Salviati fut maintenue jusqu'à récemment, avant d'être corrigée en faveur de Sabatini ${ }^{12}$.

Figure 4

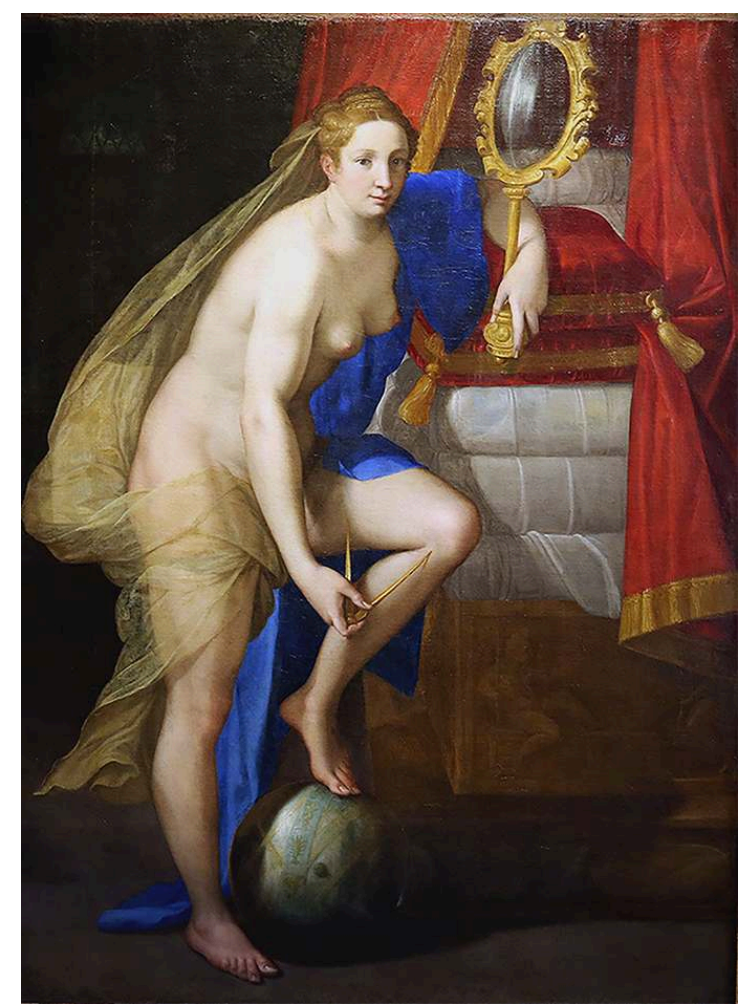

Lorenzo Sabatini, Le «mariage parfait ». Turin, Galleria Sabauda.

Phot. Michele Danieli. (c) Galleria Sabauda, Turin.

L'interprétation du sujet a subsisté plus longtemps encore : déjà identifié comme une Géométrie lors de sa vente au prince Eugène de Savoie, c'est toujours ce qu'indique le cartel de la Galleria Sabauda. Dans l'Iconologie de Cesare Ripa, la Géométrie est décrite comme une «Femme, qui tient dans une main le Plomb et dans l'autre un Compas », ou bien une « Femme, qui tient un Compas dans la main droite et dans la main gauche une Équerre $^{13} »$. Comme on peut le voir, il n'y est fait aucune allusion au miroir qui, dans notre tableau, occupe une place trop importante pour être négligé. On ne peut pas non plus admettre les hypothèses alternatives de Luisa Mortari, qui propose d'identifier la figure avec la Symétrie, ou de Daniele Benati, qui voudrait y reconnaître la peinture 
représentant la «Justice qui se regarde dans le miroir » d'un palais bolonais citée par un manuscrit datant du XvIII ${ }^{\mathrm{e}}$ siècle ${ }^{14}$.

Personne ne s'est interrogé toutefois sur la raison pour laquelle, derrière une figure allégorique qui devrait d'une manière ou d'une autre avoir un lien avec les mathématiques ou les sciences géométriques, apparaissait un grand lit. Il semble évident qu'il ne s'agit pas d'un détail négligeable de la composition, car il est placé de manière très visible et est décrit dans ses moindres détails. Pour dissiper ce doute, il faut reconsidérer l'identification de la figure féminine et de ses attributs. Si l'on se rapporte au texte de Ripa, on y trouve la description de l'« CEuvre parfaite » : « Femme, qui tient un Miroir de la main droite, \& de la gauche une Elquierre, \& un Compas ${ }^{15}$. » La gravure correspondant à l'« CEuvre parfaite » dans la première édition illustrée de l' Iconologia (1603) est très proche du tableau de Sabatini.

Il semble donc possible d'imaginer que le tableau de la Galleria Sabauda ait été peint à l'occasion d'un mariage, et qu'il représente le vœu d'une parfaite union conjugale. Et pour opérer le déplacement de la signification de l'« CEuvre parfaite» dans la sphère matrimoniale, quoi de mieux qu'un lit? Dans notre cas, bien qu'il soit placé de manière très visible, il s'agit seulement de l'évocation d'un lit dont on ne voit qu'une petite partie : un lit nuptial luxueux, très élevé, et même composé de trois matelas - allusion à des plaisirs conjugaux confortables. Du reste, on ne pourrait expliquer autrement d'autres détails du tableau qui ne sont pas secondaires, comme les petits amours représentés en bas-relief sur la base du lit ni, surtout, la nudité de la femme, dont le texte de Ripa ne fait aucune mention.

Dans notre dernier tableau, le rôle du lit est totalement différent de ceux qu'il endossait dans les tableaux précédents. Il s'agit du Portrait d'enfant de Lavinia Fontana (1552-1614), conservé à la Pinacothèque de Bologne (fig. 5), qui, pour des raisons stylistiques, a été daté autour de 1583 , et qui est donc approximativement contemporain des tableaux étudiés précédemment. Bien que Lavinia soit une grande spécialiste de ce genre, ce portrait est un cas isolé dans sa production, et bien qu'il n'ait jamais quitté Bologne, il n'a été attribué à cette artiste que dans la seconde moitié du $\mathrm{xx}^{\mathrm{e}}$ siècle. 
Figure 5

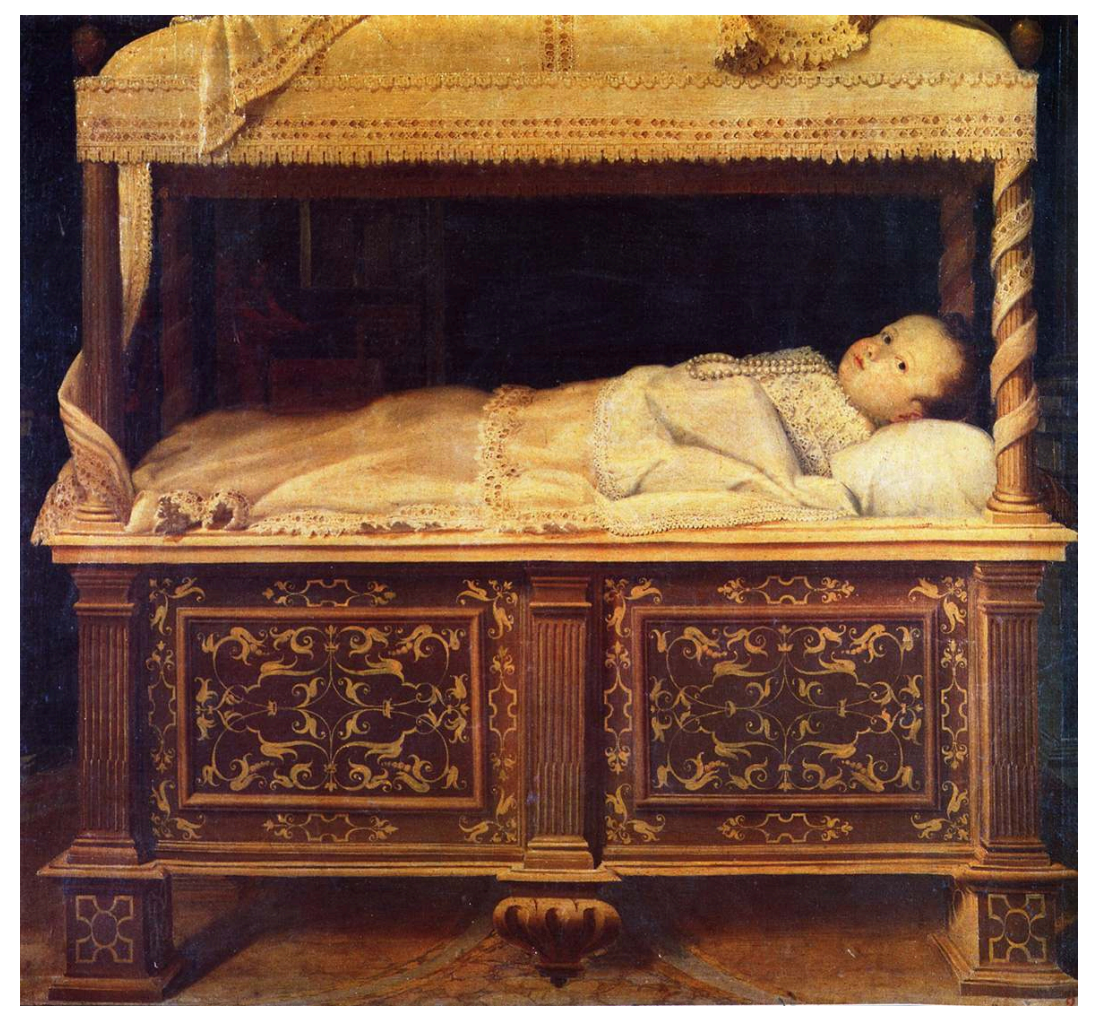

Lavinia Fontana, Portrait d'enfant. Bologne, Pinacothèque nationale.

(C) MIBACT - Archivio fotografico Pinacoteca Nazionale, Bologna.

18 Il fut donné à la ville de Bologne en 1761 par le comte Giulio Bianchetti, avec un autre tableau représentant une dame de sa famille, la poétesse Giovanna Bianchetti, qui vécut au XIV ${ }^{e}$ siècle : l'enfant appartint probablement lui aussi à la même famille, mais nous ne connaissons pas son nom. Au moment de la donation, il était associé à une incroyable attribution à Léonard qui survécut jusqu'à la fin du XIx ${ }^{\mathrm{e}}$ siècle ; par la suite, on trouve les noms de Federico Barocci (1528-1612) et de Santi di Tito (1536-1603) dans le catalogue du musée ${ }^{16}$.

19 En réalité, la solution au problème apparaissait déjà dans une note de l'édition de la Felsina pittrice de Malvasia du 1841: "un tableau attribué à Lavinia figurant un Bébé royal qui repose dans un petit lit ornementé, enveloppé dans des langes et portant un collier ${ }^{17}$ ». La richesse du berceau et des étoffes avait fait penser à un bébé royal qui, pourtant, à Bologne (ville sans cour, comme nous l'avons dit en introduction), n'aurait pas pu naître. Il faut toutefois reconnaître que même des nouveau-nés plus nobles, comme Federico della Rovere, tel qu'il est portraituré par Federico Barocci en 1605, ou Leopoldo de' Medici par Tiberio Titi (1578-1637) en 1617, tous les deux au palais Pitti, ne disposent pas de berceaux aussi monumentaux.

20 À la différence de ce qui se passe dans les tableaux de Samacchini, Calvaert et Sabatini, Lavinia montre ici son intention de dépeindre un véritable meuble, témoignage d'un luxe tangible, fait de matériaux durables et œuvre d'un artisanat savant, sans la moindre trace de rêverie érotique ou allégorique, ni sculptures complexes ou ornements dorés. Le berceau consiste en une structure compacte, avec trois pilastres cannelés à chapiteaux simples qui encadrent deux panneaux ornés de marqueteries 
végétales. Quatre fines colonnes supportent un petit baldaquin recouvert d'une étoffe brodée ; dans la partie haute court un ruban d'où pendent des breloques.

21 La préciosité de l'ensemble apparaît dans le trousseau de draps brodés, qui constituaient un élément important de la richesse domestique, aujourd'hui totalement perdue et oubliée. Notre tableau manifeste la volonté de signifier une condition basée sur une richesse solide, bien enracinée dans les conventions de l'époque, expression d'une petite noblesse très attentive à souligner tous les signes de distinction sociale qu'il était possible d'inscrire dans ce contexte: un collier de perles, une demeure si spacieuse qu'il est possible de consacrer une pièce entière au seul petit lit, et naturellement, un trousseau de la meilleure qualité. Et aussi le portrait d'un enfant, certainement, mais surtout le portrait d'un lit bolonais de la fin du Cinquecento.

\section{NOTES}

1. - AJMAR-WOLLHEIM, Marta, DENNIS, Flora (éd.). At Home in Renaissance Italy. Londres : V \& A Publications, 2006 ; CAVALLO, Sandra, STOREY, Tessa. Healthy Living in Late Renaissance Italy. Oxford: Oxford University Press, 2013, p. 113-144 (« A Good Night's Sleep »); voir aussi l'étude de cas traitée dans LEZOTTE, Annette. The Home Setting in Early Netherlandish Paintings: A Statistical and Iconographical Analysis of Fifteenth-and Early Sixteenth-century Domestic Imagery. Lewiston: Edwin Mellen Press, 2008 ; on peut aussi citer un ouvrage de vulgarisation : FRUGONI, Chiara. Vivere nel Medioevo. Donne, uomini e soprattutto bambini. Bologne: Il Mulino, 2017, p.13-38 («Felicità domestica, il letto e i suoi usi »).

2. - La célèbre Annonciation de Ludovico Carrache, conservée à la Pinacothèque de Bologne, ne fait pas exception (BROGI, Alessandro. Ludovico Carracci (1555-1619). Ozzano Emilia: Tipoarte, 2001, p. 221-223).

3. - Inv. 4637, huile sur toile, $249 \times 178 \mathrm{~cm}$. Il entra dans les collections en 1797 à l'occasion des saisies révolutionnaires, avec une attribution à Martin Fréminet; BREJON de LAVERGNÉE, Arnauld, THIÉBAUT, Dominique. Catalogue sommaire illustré des peintures du musée du Louvre. Italie, Espagne, Allemagne, Grande-Bretagne et divers. Paris: RMN, 1979, p. 234 ; WINKELMANN, Jürgen. «Orazio Samacchini». Dans FORTUNATI PIETRANTONIO, Vera. Pittura bolognese del'500. Casalecchio di Reno : Grafis, 1986, p. 636 («fondamentale per il percorso di Orazio Samacchini »); FOUCART-WALTER, Élisabeth, HABERT, Jean, LOIRE, Stéphane, SCAILLIÉREZ, Cécile, THIÉBAUT, Dominique. Catalogue des peintures italiennes $d u$ Musée du Louvre. Catalogue sommaire. Paris: Gallimard, 2007, p. 99. Voir dans la base Joconde : notice 0PE027031.

4. - « Ut primum alatis tegeti magalia plantis, / Aeneam fundantem arces ac tecta novantem / conspicit: atque illi stellatua iaspide fulva / ensis erat tyrioque ardebat murice laena, / demissa ex umeris, dive quae munera Dido / fecerat et tenui telas discreverat auro » (VIRGILE. Énéide. IV, § 259-264).

5. - « Enea che, spogliandosi per andare a dormire con Dido, è sopragiunto da Mercurio » (VASARI, Giorgio. Le vite de' più eccellenti pittori, scultori, e architettori. Florence : Giunti, 1568, V, p. 545) ; la copie a été publiée par Voss, Hermann. «Ein wiedergefundenes Bild des Daniele da Volterra ». Kunstchronik, 1922-1923, XXIV, p. 375-37; voir aussi CIARDI, Roberto Paolo, MORESCHINI, Benedetta. Daniele Ricciarelli. Da Volterra a Roma. Milan: Motta, 2004, p. 232-240 ; HOCHMANN, Michel. «Les dessins et les peintures de Fulvio Orsini et la collection Farnèse ». Mélanges de l'École 
française de Rome. Italie et Méditerranée, 1993, CV, 1, p. 76, n. 12 ; PAGLIANO, Éric. L'Atelier de l'œuvre. Dessins italiens du musée Fabre. Courtrai/Montpellier : Snoeck/musée Fabre, 2013, p. 139-140.

6. - LUCIEN. «Hérodote ou Aétion », § 4-6; pour une autre interprétation : LOVISETTO, Luana. «La pittura descritta: ekphrasis e riconversioni ecfrastiche nel Quattrocento e Cinquecento». Dans CENTANNI, Monica (éd.). L'originale assente. Introduzione allo studio della tradizione classica. Milan: Mondadori, 2005, p. 393-399. Outre les dessins de Raphaël à l'Albertina (Vienne) et au musée Teylers (Haarlem, Pays-Bas), l'exemple le plus célèbre est la fresque du Sodoma à la Farnésine (Rome, 1519) dans laquelle, sur la gauche, apparaît un lit magnifique, très similaire à son contemporain (1515) conservé au Musée lorrain (Nancy), étudié par Pierre-Hippolyte Pénet. Voir dans ce numéro: BARBIER, Muriel et PÉNET, Pierre-Hippolyte. «Le lit du duc Antoine de Lorraine et de la duchesse Renée de Bourbon : une nouvelle vie grâce au numérique ", In Situ [En ligne], 40|2019, mis en ligne le 15 septembre 2019, consulté le 30 septembre 2019. URL: http://journals.openedition.org/insitu/24089.

7. - LUCIEN. «Hérodote ou Aétion », § 6 (dans la traduction d'Eugène Talbot. Paris : Hachette, 1912).

8. - LABORIE, Séverine. " "Mercure ordonne à Énée d'abandonner Didon" d'Orazio Samacchini : une œuvre dévoilée par sa restauration ». La Revue des musées de France, 2008, LVIII, 4, p. 70-78.

9. - Inv. 1938 E 236, huile sur toile, $145 \times 110 \mathrm{~cm}$. Attribuée à un anonyme flamand, elle fut prudemment attribuée à Calvaert par BRÉJON de LAVERGNÉE, Arnauld. Dijon, Musée Magnin. Catalogue des tableaux et dessins italiens (xve - XIxe siècles). Paris : Réunion des musées nationaux, 1980, p. 120-121, et avec plus de conviction par BENATI, Daniele. «Lorenzo Sabbatini: quadri 'con donne nude' ». Dans BÉGUIN, Sylvie, DI GIAMPAOLO, Mario. Scritti di storia dell'arte in onore di Jürgen Winkelmann. Naples: Paparo, 1999, p. 57 n. 22 ; sur la chronologie, voir DANIELI, Michele. "Pittura erotica tra Bologna e Praga: aggiunte a Denys Calvaert e Dirck de Quade van Ravesteyn ». Czech and Slovak Journal of Humanities, 2016, 3, p. 39-52, voir le site : http://www2.culture.gouv.fr/ public/mistral/joconde_fr?

ACTION=RETROUVER\&FIELD_98=TOUT\&VALUE_98=calvaert\%20\&NUMBER=3\&GRP=0\&REQ=\%28\%28calvaert\%29\%20\%3aTOUT\%20\%29\&

10. - Non exposé. Inv. $\mathrm{n}^{\circ} 65.2211$.

11. - TITE-LIVE. Ab urbe condita, I, 58 : « Vestigia viri alieni, Collatine, in lecto sunt tuo ».

12. - L'attribution à Salviati fut reprise par GABRIELLI, Noemi. Galleria Sabauda. Maestri italiani. Turin : Ilte, 1971, p. 222, et par MORTARI, Luisa. Francesco Salviati. Rome : De Luca, 1992, p. 121 ; sur l'histoire du tableau, voir DANIELI, Michele. «Lorenzo Sabatini e Guido Reni. Per la provenienza di due dipinti della Galleria Sabauda ». Studi Piemontesi, 2017, XLVI, 2, p. 519-526.

13. - "Donna, che tenga con una mano un Perpendicolo, \& con l'altra un Compasso"; " Donna, che con la destra tiene un Compasso, \& con la sinistra un Triangolo ». RIPA, Cesare. Iconologia. Rome : Heredi di Gio. Gigliotti, 1593, p. 103.

14. - L'écrivain bolonais Marcello Oretti la situe au palais Ratta à Bologne : BENATI, Daniele. « Lorenzo Sabbatini: quadri "con donne nude" ». Dans BÉGUIN, Sylvie, DI GIAMPAOLO, Mario, NARCISI, Pietro (éd.). Scritti di storia dell'arte in onore di Jürgen Winkelmann. Naples : Paparo, 1999, p. 51, mais la description ne correspond pas, et vers 1770, au moment où Oretti écrit, le tableau se trouvait déjà à Vienne depuis un demi-siècle.

15. - RIPA, Cesare. Iconologie, ou la science des emblèmes, devises, etc., qui apprend à les appliquer, dessiner et inventer. Trad. Jean Baudoin. Amsterdam : Adrian Braakman, 1698, CXI, fig. 109, p. 162, texte p. 165 (consultable sur Gallica). Il manque, il faut l'admettre, une référence explicite au globe terrestre sur lequel la femme pose le pied.

16. - Le nom de Léonard fut repris, entre autres, par LANZI, Luigi. Storia pittorica della Italia. Bassano : Remondini, 1795-1796, I, p. 107 ; on doit le changement définitif d'attribution en faveur de Lavinia à GHIRARDI, Angela. "Una pittrice bolognese nella Roma del Seicento: Lavinia Fontana ». Il Carrobbio, 1984, X, p. 158-161 ; CANTARO, Maria Teresa. Lavinia Fontana bolognese: "pittora singolare", 1552-1614. Milan: Jandi Sapi, 1989, p. 135-136; DERN, Alexandra. 
«Beobachtungen zu Kinderbildnissen in Cuvre Lavinia Fontana ». Dans DOMBROWSKI, Damian (éd.). Zwischen den Welten. Beiträge zur Kunstgeschichte für Jürg Meyer zur Capellen. Weimar : VDG, 2001, p. 128-136 ; MURPHY, Caroline. Lavinia Fontana. A painter and her patrons in sixteenth-century Bologna. New Haven: Yale University Press, 2002, p. 175-176; GRAZIANI, Irene. "Lavinia Fontana ». Dans BENTINI, Jadranka, CAMMAROTA, Gian Piero, MAZZA, Angelo, SCAGLIETTI KELESCIAN, Daniela, STANZANI, Anna (éd.). Pinacoteca Nazionale di Bologna. Catalogo generale. 2. Da Raffaello ai Carracci. Venise : Marsilio, 2006, p. 209-210.

17. - Notre traduction. MALVASIA, Carlo Cesare. Felsina pittrice. Vite de'pittori bolognesi [1678]. Éd. Giampietro Zanotti. Bologne : Guidi all'Ancora, 1841, p. 178, n. 5.

\section{RÉSUMÉS}

Quelques exemples issus de la peinture bolonaise de la fin $\mathrm{du} \mathrm{XVI}^{\mathrm{e}}$ à la fin $\mathrm{du} \mathrm{XVII}^{\mathrm{e}}$ siècle illustrent les différentes significations que l'on peut attribuer au lit dans divers contextes narratifs. Le lit peut devenir une grande scène sur laquelle Orazio Samacchini réécrit un épisode célèbre de l' Énéide en en accentuant son caractère sensible et intime. Les personnages féminins des peintures de Denis Calvaert vivent des aventures tragiques, mais avec diverses nuances: la rectitude morale de Lucrèce, l'imprudence de Sémélé imposent des mises en scène adéquates, et l'artiste choisit pour chacune le lit le plus adapté. Le lit que Lorenzo Sabatini inclut dans son tableau possède quant à lui une signification purement symbolique, que le peintre emploie pour enrichir une représentation allégorique. L'absence d'une cour et d'un centre de pouvoir a privé Bologne, à l'époque moderne, de la production de produits et de meubles destinés à la résidence d'un souverain. Il faut donc rechercher le paradigme du mobilier de luxe dans la peinture maniériste florentine et romaine ou dans les estampes flamandes, autant d'éléments à la portée des artistes, plutôt que dans des objets royaux réels. C'est ce que démontre de manière évidente un tableau de Lavinia Fontana, un véritable " portrait de lit » : le spectacle qu'il offre d'une aisance concrète le distingue des tableaux étudiés précédemment.

Some examples taken from the paintings executed at Bologna from the end of the sixteenth century to the end of the seventeenth illustrate the different meanings that can be attributed to the bed in different narrative contexts. The bed can become a major theatrical scene on which Orazio Samacchini rewrites a famous episode from the Aeneid, giving special emphasis to its sensitive and intimate character. The female figures in the paintings of Denis Calvaert live tragic adventures, but with varying outcomes. The moral rectitude of Lucretia or the recklessness of Semele impose appropriate settings and for each one, the artist choses the bed that is most suitable. The bed that Lorenzo Sabatini includes in his painting has purely symbolic significance which the painter uses in order to enrich his allegorical representation. The absence of a court and a centre of private power at Bologna during the early modern period means that it was not a centre for the production of furniture for a sovereign's residence. So, the paradigm of luxury furniture is to be looked for in the mannerist painting of Florence or Rome or in Flemish engravings, articles that were more accessible to artists than the real royal objects. This is clearly demonstrated in a painting by Lavinia Fontana, which is a proper 'portrait' of a bed. The spectacle it offers of real affluence distinguishes this painting from the others examined here. 
INDEX

Keywords : bed, cradle, Bologna, sixteenth century, Cinquecento, Seicento, seventeenth century, painting, iconography of Bologna, Orazio Samacchini, Daniele da Volterra, Denis Calvaert, Lorenzo Sabatini, Cesare Ripa, Iconologia, Lavinia Fontana

Mots-clés : Lit, berceau, Bologne, XVIe siècle, Cinquecento, Seicento, XVIIe siècle, peinture, iconographie, bolonais, Orazio Samacchini, Daniele da Volterra, Denis Calvaert, Loernzo Sabatini, Cesare Ripa, Iconologia, Lavinia Fontana

\section{AUTEUR}

\section{MICHELE DANIELI}

Università di Bologna, chercheur associé micheledanieli@hotmail.com 